\title{
Semiclassical Modeling of Isotropic Non-Heisenberg Magnets for Spin $S=1$ and Linear Quadrupole Excitation Dynamics
}

\author{
Yousef Yousefi and Khikmat Kh. Muminov \\ S.U. Umarov Physical-Technical Institute, Academy of Sciences of Republic of Tajikistan, Aini Avenue 299/1, \\ Dushanbe, Tajikistan
}

Correspondence should be addressed to Yousef Yousefi; yousof54@yahoo.com

Received 25 January 2013; Accepted 10 March 2013

Academic Editor: Ali Hussain Reshak

Copyright ( 2013 Y. Yousefi and K. Kh. Muminov. This is an open access article distributed under the Creative Commons Attribution License, which permits unrestricted use, distribution, and reproduction in any medium, provided the original work is properly cited.

Equations describing one-dimensional non-Heisenberg model are studied by use of generalized coherent states in real parameterization, and then dissipative spin wave equation for dipole and quadrupole branches is obtained if there is a small linear excitation from the ground state. Finally, it is shown that for such exchange-isotropy Hamiltonians, optical branch of spin wave is nondissipative.

\section{Introduction}

Many condensed matter systems are fully described by use of effective continuum field models. Topologically nontrivial field configurations have an important role in modeling systems with reduced spatial dimensionality [1]. Magnetic systems are usually modeled with the help of the Heisenberg exchange interaction [2-4].

However, for spin $S>1 / 2$, the general isotropic exchange goes beyond the purely Heisenberg interaction bilinear in spin operators $\vec{S}_{i}$ and includes higher order terms of the type $\left(\vec{S}_{i} \vec{S}_{j}\right)^{n}$ with $n$ up to $2 S$ [5]. Due to the spin states, the $2 S+1$ complex parameters are necessary to describe each of them, and this corresponds with the $4 S+2$ degrees of freedom. Two degrees of freedom are omitted, one because of normalization condition and the other for arbitrary phase decrease, hence $4 \mathrm{~S}$ parameters are required to completely modeled the remainder $4 S$ degrees of freedom of spin states [6].

Particularly, in case $S=1$ with the isotropic nearest neighbor exchange on a lattice is derived by use of the Hamiltonian

$$
\widehat{H}=-\sum_{i} J\left(\overrightarrow{\widehat{S}}_{i} \overrightarrow{\widehat{S}}_{i+1}\right)+K\left(\overrightarrow{\widehat{S}}_{i} \overrightarrow{\widehat{S}}_{i+1}\right)^{2}
$$

Here $\widehat{S}_{i}^{x}, \widehat{S}_{i}^{y}, \widehat{S}_{i}^{z}$ are the spin operators acting at a site $i, J$ and $K$ are, respectively, the bilinear (Heisenberg) and biquadratic exchange integrals. The model (1) has been discussed recently in connection with $S=1$ bosonic gases in optical lattices [7] and in the context of the deconfined quantum criticality $[8,9]$. Hamiltonian (1) is a special form presented in [10] and because of importance of quadrupole excitation in ferromagnetic Materials, it is considered here. This paper does not consider the antiferromagnetic and nematic states.

Considering the effects of both dipole and quadrupole branches gives a nonlinear approximation. If higher order multipole effects are considered, the approximation is more accurate but at the same time, deriving the equations is too complicated. In this paper, only the effect of quadrupole branch for Hamiltonians described by (1) is considered. Study of isotropic and anisotropic spin Hamiltonian with nonHeisenberg terms is complicated due to quadrupole excitation dynamics $[5,11,12]$. Antiferromagnetic property of this excitation in states near the ground proves the existence of it, and Dzyaloshinskii calculated the effect of this excitation [13]. Also, numerical calculations more accurately justify laboratory results if the effect of quadrupole excitation in nanoparticles $\mathrm{Fe}_{8}$ and $\mathrm{Mn}_{12}$ is considered $[14,15]$. In addition, this method may be promising for description of the multispin 
configuration of the Fe in the different ligand coordinations [16].

To calculate effect of quadrupole excitation, at first it is necessary to obtain classical equivalent of Hamiltonian (1), and then to find out the solution of spin wave, it is necessary to analyze resulted equations in case there is small linear excitation from the ground states. Therefore, the stages of process are the following.

(1) Obtaining coherent states for spin $s=1$ which are coherent states of $\mathrm{SU}(3)$ group.

(2) Calculation of average values of spin operator.

(3) Classical spin Hamiltonian equation is obtained using previously calculated values.

(4) Calculating Lagrangian equation by use of Feynman path integral over coherent states and then computing classical equations of motion.

(5) It is necessary to substitute resulted Hamiltonian in classical equations of motion to obtain nonlinear equations of magnets. Solutions of these nonlinear equations result in soliton description of magnet that is not interested here.

(6) Now, ground states of magnets calculated and then nonlinear equations are linearized above the ground states for small linear excitation.

(7) Finally, spin wave equations and dispersion equations must be calculated.

In this paper, we write coherent states in real parameters, because each parameter in this representation related to one degree of freedom. In complex parameterization, each parameter related to two or more degrees of freedom. Then in physical problems, the first representation is very helpful.

In next sections, we develop mathematical descriptions of the pervious stages and analyze these descriptions.

\section{Theory and Calculations}

In quantum mechanics, coherent states are special kinds of quantum states that their dynamics are very close to their corresponding classical system. Type of coherent state is used in problem and depends on the operators symmetry in Hamiltonian. Due to the operators symmetry in Hamiltonian (1), for more detailed description and considering all multipolar excitation of coherent states we used coherent states in SU(3) group. Vacuum state of this group is $(1,0,0)^{T}$ and coherent state for a single site in this group is introduced as [11]:

$$
|\psi\rangle=D^{1}(\theta, \phi) e^{-i \gamma \widehat{S}^{z}} e^{2 i g \widehat{Q}^{x y}}|0\rangle,
$$

where $D^{1}(\theta, \phi)$ is wigner function and $Q^{x y}$ is quadrupole moment. Two angles, $\theta$ and $\phi$, the Euler angles, determine the direction of classical spin vector in spherical coordinate system. The angle $\gamma$ determines the direction of quadrupole moment around the spin vector and parameter $g$ shows change of magnitude of spin vector and average value of quadrupole moment. Two angles $\phi$ and $\gamma$ change between 0 to $\pi$ and angle $\theta$ changes between $-\pi$ to $\pi$.
In order to derive Lagrangian from path integral, we drive the path integral from the following transition amplitude:

$$
P\left(\psi_{1}, t_{1} ; \psi, t\right)=\left\langle\psi_{1}\left|\exp \left(-\frac{i}{\bar{h}}\left(\widehat{H}\left(t_{1}-t\right)\right)\right)\right| \psi\right\rangle .
$$

Using completeness relation and doing some mathematical work, the following equation for transition amplitude obtained:

$$
\begin{aligned}
& P\left(\psi_{1}, t_{1} ; \psi, t\right) \\
& \quad=\lim _{n \rightarrow \infty} \sum_{j} \int D \mu_{j}(\psi) \exp \left(\frac{-i}{\bar{h}} \int_{t}^{t_{1}} L_{j}(\theta, \phi, g, \gamma) d \tau\right) .
\end{aligned}
$$

In the pervious equation $L$ is Lagrangian and has the following form [11]:

$$
L=\bar{h} \cos 2 g\left(\cos \theta \phi_{t}+\gamma_{t}\right)-H(\theta, \phi, g, \gamma) \text {. }
$$

$x_{t}=(\partial x / \partial t)(x=\theta, \phi)$ and $H(\theta, \phi, g, \gamma)$ is classical Hamiltonian. By use of (4) and (5) and the action stationary principle, the classical equations of motion are obtained.

When Lagrangian is obtained from path integral, another two terms appear, one is kinetic term and has "Berry phase" properties that are important in spin tunneling phenomena and the other depends on boundary condition values. In this paper, we do not consider these two terms.

Now classical equivalent of spin vector and their products must be computed so that the classical equivalent of Hamiltonian (1) is obtained. So consider the following:

$$
\vec{S}=\langle\psi|\overrightarrow{\widehat{S}}| \psi\rangle
$$

as classical spin vector, and also consider the following:

$$
\widehat{Q}^{i j}=\frac{1}{2}\left(\widehat{S}_{i} \widehat{S}_{j}+\widehat{S}_{j} \widehat{S}_{i}-\frac{4}{3} \delta_{i j} I\right)
$$

components of quadrupole moment. Because we can write any coherent state as product of single site coherent states, namely,

$$
|\psi\rangle=\prod_{i}|\psi\rangle_{i}
$$

Then spin operators in ground state of nonsingle ions Hamiltonian can be commuted in different lattices [11], so

$$
\left\langle\psi\left|\widehat{S}_{n}^{i} \widehat{S}_{n+1}^{j}\right| \psi\right\rangle=\left\langle\psi\left|\widehat{S}_{n}^{i}\right| \psi\right\rangle\left\langle\psi\left|\widehat{S}_{n+1}^{i}\right| \psi\right\rangle,
$$

where $|\psi\rangle=|\psi\rangle_{n}|\psi\rangle_{n+1}$.

The average spin values in $\mathrm{SU}(3)$ group for coherent states (2) are defined as [17]

$$
\begin{gathered}
S^{+}=e^{i \phi} \cos (2 g) \sin \theta, \\
S^{-}=e^{-i \phi} \cos (2 g) \sin \theta, \\
S^{z}=\cos (2 g) \cos \theta .
\end{gathered}
$$


And also

$$
\begin{gathered}
S^{2}=\cos ^{2}(2 g), \\
q^{2}=\sin ^{2}(2 g), \\
S^{2}+q^{2}=1 .
\end{gathered}
$$

In the pervious relation, $S^{2}$ is related to dipole moment and $q^{2}$ is related to quadrupole moment. Classical Hamiltonian can be obtained from the average calculation of Hamiltonian (1) over coherent states. The classical continuous limit of Hamiltonian in $\mathrm{SU}(3)$ group is

$$
\begin{aligned}
H=-\int \frac{d x}{a_{0}}\left(J \cos ^{2}(2 g)+K \cos ^{4}(2 g)\right. & \\
-\frac{a_{0}^{2}}{2}( & 4 g_{x}^{2} \sin ^{2}(2 g)\left(J+2 K \cos ^{2}(2 g)\right) \\
& +\cos ^{2}(2 g)\left(J+2 K \cos ^{2}(2 g)\right) \\
& \left.\left.\times\left(\theta_{x}^{2}+\phi_{x}^{2} \sin ^{2} \theta\right)\right)\right),
\end{aligned}
$$

where $a_{0}$ is length of crystal sites. The pervious classical Hamiltonian is substituted in equation of motion that obtained from the Lagrangian, and the result is classical equations of motion:

$$
\begin{gathered}
\frac{1}{\omega_{0}} \theta_{t}=-a_{0}^{2} \cos (2 g)(J+K+K \cos (4 g)) \phi_{x x} \sin \theta \\
\frac{1}{\omega_{0}} \phi_{t}=a_{0}^{2} \cos (2 g)(J+K+K \cos (4 g))\left(\phi_{x}^{2} \cos \theta+\theta_{x x} \csc \theta\right) \\
\frac{1}{\omega_{0}} g_{t}=0 \\
\frac{1}{\omega_{0}} \gamma_{t}=4 \cos (2 g)(J+K+K \cos (4 g)) \\
+\left(K \operatorname { c o s } { } ^ { 3 } ( 2 g ) \left(16 g_{x}^{2}-8 \theta_{x}^{2}-5 \phi_{x}^{2}\right.\right. \\
\left.+3 \phi_{x}^{2} \cos (2 \theta)-2 \theta_{x x}\right)+\cos (2 g) \\
\times\left(8 g_{x}^{2}(J-K)-2 J \theta_{x}^{2}+8 g_{x}^{2} K \cos (4 g)\right. \\
\left.+\frac{1}{2} J \phi_{x}^{2}(-3+\cos (2 \theta))-J \theta_{x x} \cot \theta\right) \\
+4 g_{x x} J \sin (2 g) \\
\left.+2 g_{x x} K(\sin (2 g)+\sin (6 g))\right) a_{0}^{2} .
\end{gathered}
$$

In these equations, $\omega_{0}$ is $\bar{h} a_{0}$. These equations describe nonlinear dynamics of non-Heisenberg ferromagnetic chain completely. If we omitted quadrupole excitation $(g=0)$ in the pervious equations, these equations reduced to LandauLifshitz equations. Then in comparison with Landau-Lifshitz equations, these equations are more complete and contain more degrees of freedom. Note that solutions of these equations are different forms of magnetic solitons.

In this paper, only the linearized form of (13) for small excitation above the ground states is considered. To this end,at first, classical ground states must be calculated, so in above Hamiltonian only nonderivative part is considered:

$$
H_{0}=-\int \frac{d x}{a_{0}}\left(J \cos ^{2} 2 g+K \cos ^{4} 2 g\right) .
$$

To find the smallest value of the $H_{0}$ we vary it respect to all the parameters, with the ground state is obtained at;

$$
g=0, \quad g=\frac{\pi}{2} .
$$

In this paper, only dispersion of spin wave in neighborhood of the ground states is studied. For this purpose, small linear excitations from the ground states, as shown in (15), are defined:

$$
2 g \longrightarrow \pi+g
$$

In this situation, the linearized classical equations motion are

$$
\begin{gathered}
\frac{1}{\omega_{0}} \theta_{t}=-a_{0}^{2}(J+2 K) \phi_{x x} \\
\frac{1}{\omega_{0}} \phi_{t}=a_{0}^{2}(J+2 K) \theta_{x x}, \\
\frac{1}{\omega_{0}} g_{t}=0 \\
\frac{1}{\omega_{0}} \gamma_{t}=-4(J+2 K) .
\end{gathered}
$$

Consider functions $\theta$ and $\phi$ as plane waves to obtain dispersion equation:

$$
\begin{aligned}
& \phi=\phi_{0} e^{i(\omega t-k x)}+\bar{\phi}_{0} e^{-i(\omega t-k x)}, \\
& \theta=\theta_{0} e^{i(\omega t-k x)}+\bar{\theta}_{0} e^{-i(\omega t-k x)} .
\end{aligned}
$$

Substitution of these equations in (17) results in dispersion equation of spin wave near the ground states.

$$
\begin{gathered}
\omega_{1}^{2}=(J+2 K)^{2} a_{0}^{2} k^{4} \omega_{0}^{2}, \\
\omega_{2}=-4(J+2 K) \omega_{0} .
\end{gathered}
$$

It is evident from (19) that in addition to the dispersion acoustic branch, there exist nondispersion optical branches which is related to the dipole and quadrupole excitations.

\section{Conclusion}

In this paper, describing equations of one-dimensional isotropic non-Heisenberg Hamiltonians are obtained using realparameter coherent states. It is shown that both dipole and 
quadrupole excitations have different dispersion if there is small linear excitation from the ground state.

In addition, it is shown that for isotropic ferromagnets, the magnitude of average quadrupole moment is constant $\left(g_{t}=0\right)$ and its dynamic, is rotational dynamics around the classical spin vector $\left(\gamma_{t} \neq 0\right)$.

\section{References}

[1] N. Manton and P. Sutcliffe, Topological Solitons, Cambridge university press, New York, NY, USA, 2004.

[2] E. L. Nagaev, "Anomalous magnetic structures and phase transitions in non-Heisenberg magnetic materials," Soviet Physics, vol. 25, no. 1, pp. 31-75, 1982.

[3] E. L. Nagaev, Magnets with Nonsimple Exchange Interactions, Nauka, Moscow, Russia, 1988.

[4] V. M. Loktev and V. S. Ostrovski, "Peculiarities of the statics and dynamics of magnetic insulators with single-ion anisotropy," Low Temperature Physics, vol. 20, no. 1, article 775, 26 pages, 1994.

[5] B. A. Ivanov, A. Yu. Galkin, R. S. Khymyn, and A. Yu. Merkulov, "Nonlinear dynamics and two-dimensional solitons for spin1 ferromagnets with biquadratic exchange," Physical Review $B$, vol. 77, no. 6, Article ID 064402, 11 pages, 2008.

[6] V. S. Ostrovskii, "Nonlinear dynamics of highly anisotropic spin-1 magnetic materials," Journal of Experimental and Theoretical Physics, vol. 64, no. 5, p. 999, 1986.

[7] A. Imambekov, M. Lukin, and E. Demler, "Spin-exchange interactions of spin-one bosons in optical lattices: Singlet, nematic, and dimerized phases," Physical Review A, vol. 68, no. 6, Article ID 063602, 24 pages, 2003.

[8] K. Harada, N. Kawashima, and M. Troyer, "Dimer-quadrupolar quantum phase transition in the quasi-one-dimensional heisenberg model with biquadratic interaction," Journal of the Physical Society of Japan, vol. 76, Article ID 013703, 4 pages, 2007.

[9] T. Grover and T. Senthil, "Quantum spin nematics, dimerization, and deconfined criticality in quasi-1D spin-one magnets," Physical Review Letters, vol. 98, Article ID 247202, 4 pages, 2007.

[10] N. Papanicolaou, "Unusual phases in quantum spin-1 systems," Nuclear Physics B, vol. 305, no. 3, pp. 367-395, 1988.

[11] O. K. Abdulloev and K. K. Muminov, "Semiclassical description of anisotropic magnets acted upon by constant external magnetic fields," Physics of the Solid State, vol. 36, no. 1, pp. 93-97, 1994.

[12] A. Y. Fridman, O. A. Kosmachev, and B. A. Ivanov, "Spin nematic state for a spin $S=3 / 2$ isotropic non-Heisenberg magnet," Physical Review Letters, vol. 106, Article ID 097202, 2011.

[13] I. E. Dzyaloshinskii, "External magnetic fields of antiferromagnets," Solid State Communications, vol. 82, no. 7, pp. 579-580, 1992.

[14] A. Garg, "Spin tunneling in magnetic molecules: quasisingular perturbations and discontinuous SU(2) instantons," Physical Review B, vol. 67, Article ID 054406, 13 pages, 2003.

[15] M. S. Foss-Feig and J. R. Friedman, "Geometric-phase-effect tunnel-splitting oscillations in single-molecule magnets with fourth-order anisotropy induced by orthorhombic distortion," Europhysics Letters, vol. 86, no. 1, article 27002, 2009.

[16] M. Matusiewicz, M. Czerwinski, J. Kasperczyk, and I. V. Kityk, "Description of spin interactions in model $\left[\mathrm{Fe}_{6} \mathrm{~S}_{6}\right]^{4+}$ supercluster," Journal of Chemical Physics, vol. 111, no. 14, pp. 6446-6455, 1999.
[17] V. G. Makhankov, M. A. Granados, and A. V. Makhankov, "Generalized coherent states and spin $S \geq 1$ systems," Journal of Physics A, vol. 29, no. 12, 2005. 

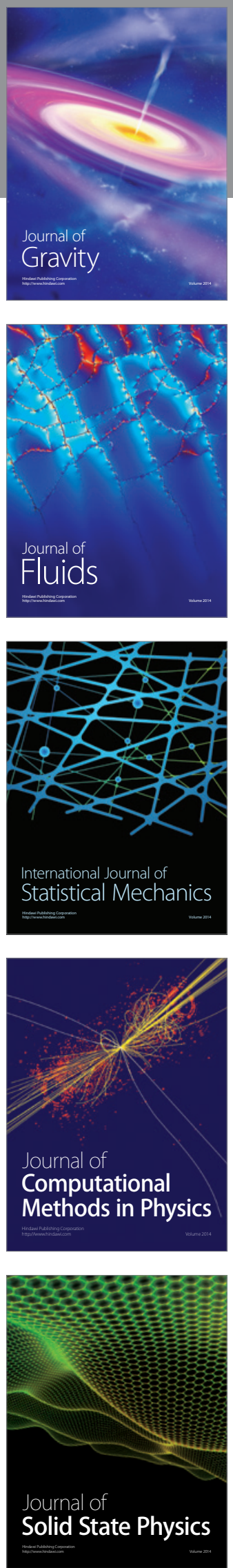

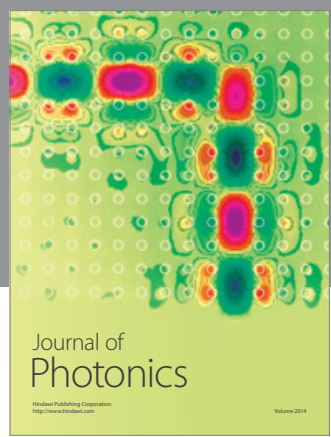

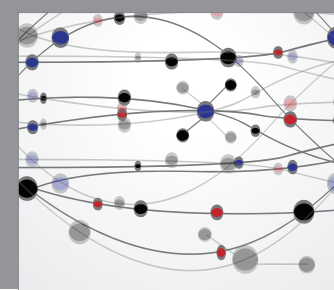

The Scientific World Journal

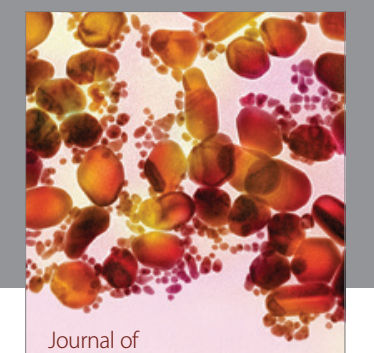

Soft Matter
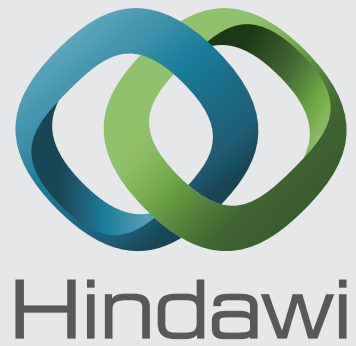

Submit your manuscripts at

http://www.hindawi.com
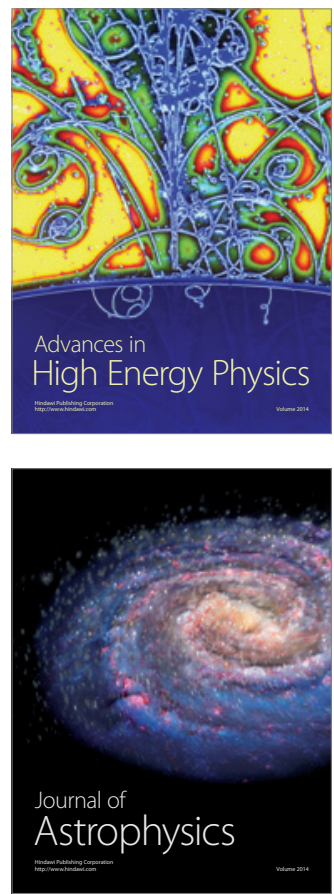
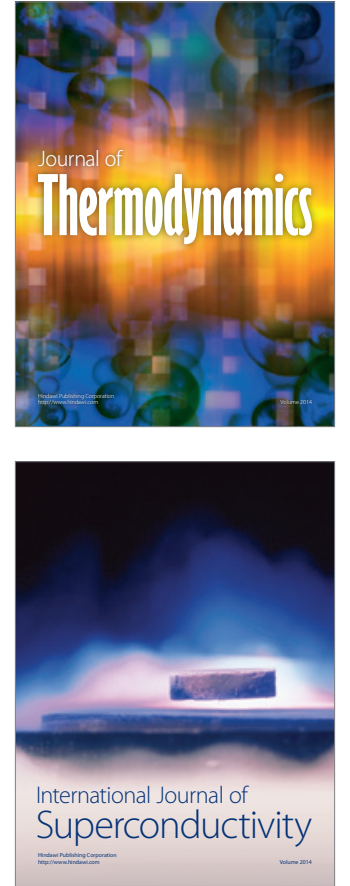
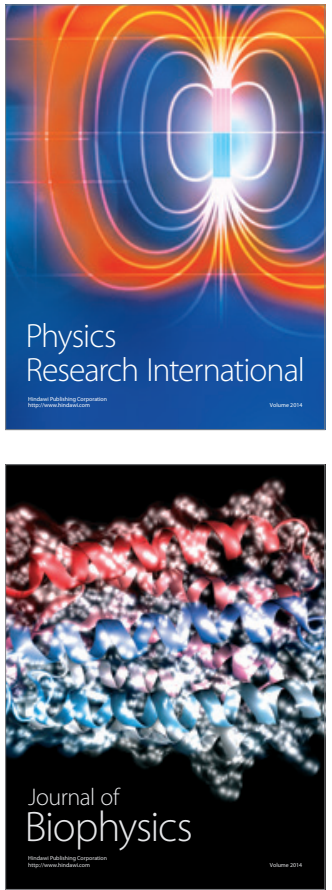
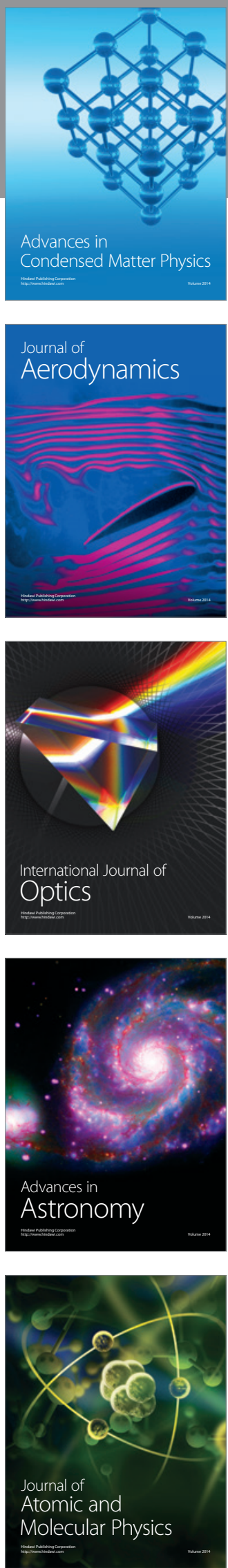